\title{
Analysis of Spectrogram Parameter Organization Applied to the Characterization of Atrial Fibrillation
}

\author{
C Vayá ${ }^{1}$, JJ Rieta $^{2}$ \\ ${ }^{1}$ Innovation in Bioengineering, Universidad de Castilla-La Mancha, Spain \\ ${ }^{2}$ Biomedical Synergy, Universidad Politécnica de Valencia, Spain
}

\begin{abstract}
Atrial Fibrillation $(A F)$ is the most common arrhythmia encountered at advanced age. The possibility of applying signal analysis to the electrocardiogram (ECG) in order to distinguish between terminating and non-terminating episodes of $A F$ would be very helpful in the regular clinical practice. In this work, an analysis of spectrogram parameters organization of the Atrial Activity $(A A)$ is carried out in order to classify between terminating and nonterminating AF episodes. The main peak frequencies, second largest peak frequencies and their respective peak magnitudes are extracted using cubic spline fitting and several numerical series are constructed from them. The independent samples mean comparison of their Sample Entropy shows five of the constructed sequences to be relevant in the characterization of AF. The bilateral significance obtained by the Student's $t$ test is less than 0.005 in five of the eight analyzed parameters.
\end{abstract}

\section{Introduction}

Atrial Fibrillation (AF) is the most common arrhythmia encountered at advanced age. The prevalence of AF remains lower than $1 \%$ among the general population, but it increases considerably from sixty years old [1]. When the AF terminates spontaneously it is classified as paroxysmal AF. On the contrary, when it sustains if no electrical or pharmacologic cardioversion is applied it is referred as persistent $\mathrm{AF}$ [1], which frequently results in permanent AF $[1,2]$. Permanent AF is closely related to a rising probability of suffering embolisms and these might provoke strokes [1]. Therefore, the possibility of distinguish between paroxysmal and persistent $\mathrm{AF}$ by applying signal analysis to the electrocardiogram (ECG) is of great interest in the regular clinical practice. In this sense, recent studies have been based on spectral analysis techniques to characterize AF episodes [3]. Furthermore, time-frequency techniques have also been used to characterize $\mathrm{AF}$ using the surface ECG [4]. The suitability of spectral analysis of AF episodes requires the previous separation of the Atrial Activity (AA) from the rest of cardioelectric signals [5]. This involves using nonlinear signal processing techniques as average beat substraction (ABS) [6] or independent component analysis (ICA) [7].

In this work, an analysis of spectrogram parameters organization of the AA is carried out with the aim to classify between terminating and non-terminating AF episodes. The analysis of these parameters is made in terms of organization of their temporal sequences. The organization is measured by using an entropy estimator, namely Sample Entropy $(S a m p E n)[8,9]$. Entropy estimators have already been used in the characterization of biomedical signals different from ECG [10]. More recent studies were centered in the organization of the main peak frequency and spectral concentration sequences for different timefrequency distributions of AA [11]. The innovation of the present essay lies, on the one hand, in the application of cubic spline fitting to the estimated spectra for a more accurate extraction of spectral parameters and, on the other hand, in the utilization of a higher number of direct and derived parameters.

\section{Database}

The signal database consisted of 30 surface ECG recordings of AF episodes which were properly annotated by cardiologists. Approximately half of the them were marked as paroxysmal $\mathrm{AF}$ and the rest as persistent $\mathrm{AF}$. The former half is subsequently refereed as the T-group and the latter as the $\mathrm{N}$-group. These recordings were of one minute in length and were previously extracted from 24-hours onelead Holter recordings of AF patients. The original sampling rate $\left(f_{s}\right)$ of the Holter systems was 128 samples per second, but ECG recordings were interpolated by a factor of 8 so that a $f_{s}$ equal to 1024 resulted. The resultant time-domain higher resolution allowed us to obtain a better cancellation of the QRS complex and a higher length of parameter sequences. In the case of the T-group patients, AF episode terminates one second after the end of the oneminute registration. On the contrary, the termination of $\mathrm{AF}$ 
episode did not occur during the whole observation time in the $\mathrm{N}$-group patients.

\section{Methods}

As a previous step, the AA had to be separated from the rest of the cardioelectric signal. To do such a think, we opted to use ABS [6] because this technique works efficiently with one-lead ECG. On the contrary, techniques based on the spatial diversity of multi-lead systems, such as blind source separation, need a higher number of leads in order to be correctly applied [7, 12]. Therefore, they were discarded because of constraints of the signal database.

After the obtention of AA signals, their spectrograms were calculated using Hamming windows of 4096 samples in length and $97 \%$ overlap. In order to facilitate the extraction of spectrogram parameters, cubic spline fitting was applied to each of the Fourier transforms composing the spectrogram. The cubic spline model obtained the best fitting in comparison with gaussian, polynomial, rational, Weibull, power and exponential models. A cubic spline is a piecewise function of polynomials which all have degree at most three [13, 14]. The cubic spline fitting curve from the original data was interpolated so that the resulting frequency increment was $0.01 \mathrm{~Hz}$. In this way, the peaks of the spectrogram were calculated more accurately. Only the main peak frequency $\left(f_{p 1}\right)$, the second largest peak frequency $\left(f_{p 2}\right)$, and their respective peak magnitudes $\left(A_{1}\right.$ and $A_{2}$ ) were extracted for later use. The fitting and extraction process is described in Fig. 1.

Four numerical series were directly constructed from the aforementioned parameters while other four series were derived from them as mathematical relations. The first of the derived parameter is the normalized distance between $f_{p 1}$ and $f_{p 2}$, which is expressed as:

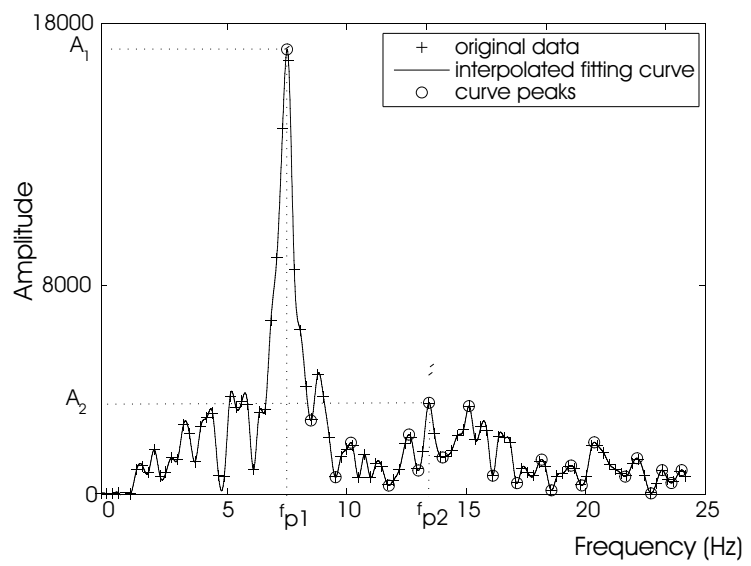

Figure 1. Fitting and extraction process of spectral parameters

$$
\Delta f_{p}=\frac{\left(f_{p 1}-f_{p 2}\right)}{f_{p 1}}
$$

The second of the derived parameters is the normalized amplitude of the second largest peak, which is defined as:

$$
\bar{A}_{2}=\frac{A_{2}}{A_{1}}
$$

The deviation of the main and second peak magnitudes from their respective expected values are also computed:

$$
\begin{aligned}
& d_{1}=f_{p 1}-\boldsymbol{E}\left(f_{p 1}\right) \\
& d_{2}=f_{p 2}-\boldsymbol{E}\left(f_{p 2}\right)
\end{aligned}
$$

where $\boldsymbol{E}(\cdot)$ represents the expected value function.

Finally, the SampEn of all theses sequences is computed as a measure of mathematical organization. Both the Approximate Entropy (ApEn) [8] and the SampEn [9] measure the organization level of time-series. That is, they quantify how predictable time-series are depending on the number of times that repetitive patterns are present in them. SampEn appears as a natural evolution of $A p E n$ with the aim to reduce the bias of this estimator [9]. The definition of SampEn can be carried out as follows:

Let $\mathrm{x}[\mathrm{n}]$ be a time-series of length $N$. The distance between any two patterns of the series, $X_{m}(i), X_{m}(j)$, of length $m$ is defined as:

$$
d\left[X_{m}(i), X_{m}(j)\right]=\max (|x(i+k)-x(j+k)|)
$$

Given a pattern $X_{m}(i)$, we calculate $B_{i}^{m}(r)$ as:

$$
B_{i}^{m}(r)=\frac{1}{N-m-1} B_{i}
$$

where $B_{i}$ is the number of patterns of length $m$ that fulfill $d\left[X_{m}(i), X_{m}(j)\right]<r$ con $1 \leq i, j \leq N-m, j \neq i$, and $r$ is the parameter that defines the criterion of similarity between patterns [8]. Next $B^{m}(r)$ is calculated as:

$$
B^{m}(r)=\frac{1}{N-m} \sum_{i=1}^{n-M} B_{i}^{m}(r)
$$

Defining $A_{i}^{m}(r)$ y $A^{m}(r)$ in the same way as $B_{i}^{m}(r)$ but considering now patterns of length $m+1$, SampEn is computed by the following expression:

$$
\operatorname{SampEn}(m, r)=-\ln \left\{\frac{A^{m}(r)}{B^{m}(r)}\right\}
$$

Pincus suggests using the values $m=1$ or $m=2$ and taking $r$ between 0.1 y 0.25 times the standard deviation of the time-series [8]. In our case, we have chosen $m$ equal to 2 and $r$ equal to 0.2 . 


\begin{tabular}{c|c|c|c|c} 
Variable & Sig. & Type & Mean & Std. \\
\hline$A_{1}$ & 0.004 & $\mathrm{~N}$ & 0.42042 & 0.06924 \\
& & $\mathrm{~T}$ & 0.30960 & 0.11940 \\
$A_{2}$ & 0.860 & $\mathrm{~N}$ & 0.29860 & 0.05741 \\
& & $\mathrm{~T}$ & 0.29245 & 0.12355 \\
$f_{p 1}$ & 0.001 & $\mathrm{~N}$ & 0.12256 & 0.02985 \\
& & $\mathrm{~T}$ & 0.08096 & 0.03152 \\
$f_{p 2}$ & 0.009 & $\mathrm{~N}$ & 0.21135 & 0.05547 \\
& & $\mathrm{~T}$ & 0.14858 & 0.07109 \\
$\Delta f_{p}$ & \multirow{2}{*}{0.003} & $\mathrm{~N}$ & 0.22634 & 0.04560 \\
& & $\mathrm{~T}$ & 0.15406 & 0.07444 \\
$\bar{A}_{2}$ & \multirow{2}{*}{0.523} & $\mathrm{~N}$ & 0.37014 & 0.07962 \\
& & $\mathrm{~T}$ & 0.39578 & 0.13407 \\
$d_{1}$ & 0.015 & $\mathrm{~N}$ & 0.55653 & 0.07871 \\
& & $\mathrm{~T}$ & 0.45289 & 0.13694 \\
$d_{2}$ & 0.948 & $\mathrm{~N}$ & 0.41963 & 0.09570 \\
& & $\mathrm{~T}$ & 0.41651 & 0.15862 \\
\hline
\end{tabular}

Table 1. Bilateral significance, mean and variance from the $\mathrm{t}$ of Student test applied to the SampEn for the spectrogram parameters $A_{1}, A_{2}, f_{p 1}, f_{p 2}, \Delta f_{p}, \bar{A}_{2}, d_{1}$ and $d_{2}$ of type $\mathrm{N}$ and $\mathrm{T}$ recordings.

\begin{tabular}{c|c} 
Variable & Area under ROC curve \\
\hline$A_{1}$ & $79.9 \%$ \\
$A_{2}$ & $53.1 \%$ \\
$f_{p 1}$ & $82.6 \%$ \\
$f_{p 2}$ & $76.3 \%$ \\
$\Delta f_{p}$ & $77.7 \%$ \\
$\bar{A}_{2}$ & $42.0 \%$ \\
$d_{1}$ & $76.3 \%$ \\
$d_{2}$ & $48.2 \%$ \\
\hline
\end{tabular}

Table 2. Area under ROC curve of SampEn for the spectrogram parameters $A_{1}, A_{2}, f_{p 1}, f_{p 2}, \Delta f_{p}, \bar{A}_{2}, d_{1}$ and $d_{2}$.

\section{Results}

Results of the Student's $t$ test applied to the SampEn of the numerical series are summarized in table 1. There we present the mean, variance and bilateral significance of the SampEn for the sequences of the spectrogram parameters $A_{1}, A_{2}, f_{p 1}, f_{p 2}, \Delta f_{p}, \bar{A}_{2}, d_{1}$ and $d_{2}$. Results of the test reveal that it is possible to distinguish between terminating and non-terminating AF in five of the eight parameters. These five relevant parameters are $f_{p 1}, f_{p 2}, \Delta f_{p}$, $A_{1}$, and $d_{1}$, which bilateral significances are, respectively, 0.001, 0.009, 0.003, 0.004 and 0.015. The mean SampEn in N-type signals is higher than in T-type signals for all these relevant parameters. This is consistent with the fact that when the episode is near the termination the fibrillatory waves become more organized and, in consequence, more predictable [15]. In the rest of parameters the bilateral significance obtained is higher than 0.05 , thus there are considered as irrelevant.

The significance of the analyzed parameters can be observed from the point of view of the Receiver Operating Characteristic (ROC) curves. This is shown in table 2 and figures 2 and 3, where the non-terminating case, i.e. $\mathrm{N}$ type, is considered as the positive state. The area under ROC curves always exceeds the $76 \%$ when the relevant parameters are used. On the contrary, the area under ROC curves for the non-relevant parameters does not reach $54 \%$ in any case.

In a more detailed analysis, if we attend to the Fig. 2 for parameter $\Delta f_{p}$ we see that it is possible to choose a threshold so that the $87.5 \%$ of the $\mathrm{N}$ type patients are correctly classified with a false alarm probability equal to $35.7 \%$. Concretely, the value of this threshold is 0.18062 . In the same way, we can consider a threshold equal to 0.36744 for parameter $A_{1}$ so that $87.5 \%$ of positive cases are correctly classified with a false alarm equal to $28.6 \%$. Similar

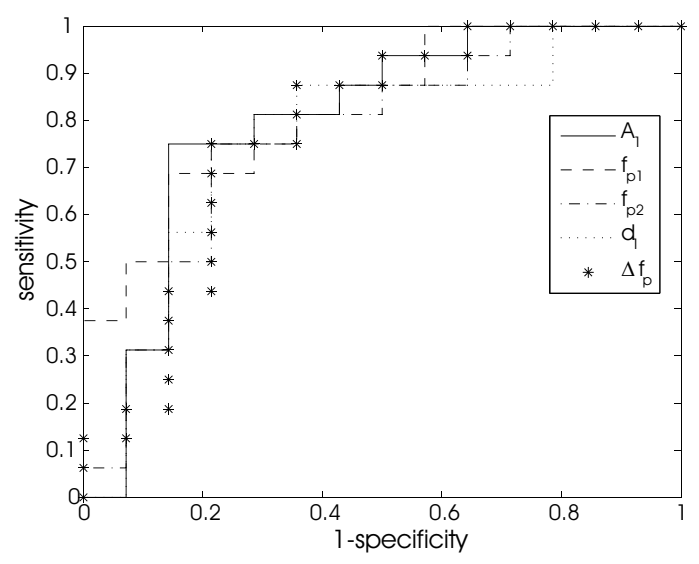

Figure 2. ROC curve of the SampEn for the spectrogram significative parameters $A_{1}, f_{p 1}, f_{p 2}, d_{1}$ and $\Delta f_{p}$.

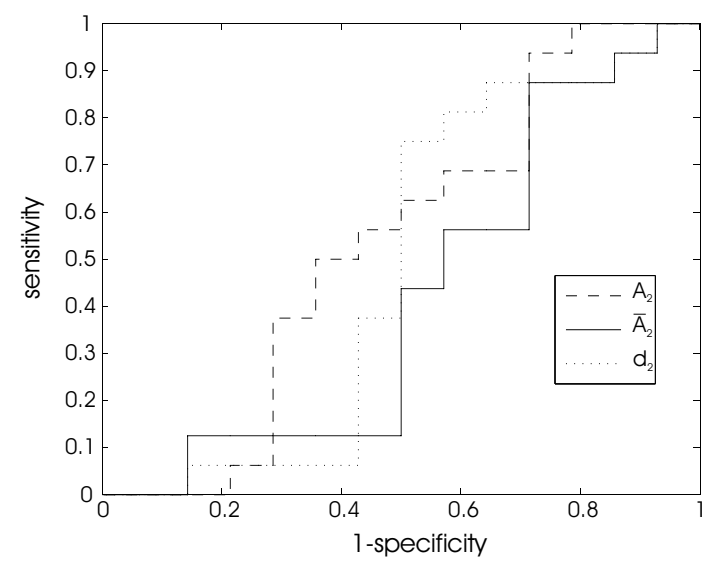

Figure 3. ROC curve of the SampEn for the spectrogram non-significative parameters $A_{2}, \bar{A}_{2}$ and $d_{2}$. 
results are obtained for the rest of relevant parameters. On the other hand, Fig. 3 illustrates that no useful decision threshold can be chosen for the rest parameters.

\section{Discussion}

The organization measure of sequences obtained from spectrogram parameters of AA signals can be used as a valid method for episode characterization. Five of the eight analyzed parameters resulted to be clinically relevant in the decision between T-type and N-type signals. The mean SampEn of the relevant parameters is higher in terminating than in non-terminating episodes, what can be seen as a higher degree of spatial organization of AF. Further research will be centered in refine the method and improve the results obtained in the present essay.

\section{Acknowledgements}

This work was partly supported by the projects 20070086 from the R+D+i Vice-rectorate of the Valencia University of Technology, GV06/299 from Consellería de Empresa, Universidad y Ciencia de la Generalitat Valenciana and TEC2007-64884 from the Spanish Ministry of Education and Science.

\section{References}

[1] Fuster V, Ryden LE, Asinger RW, et al. CC/AHA/ESC guidelines for the management of patients with atrial fibrillation. Journal of the American College of Cardiology 2001;38(4):1266/I-1266/LXX.

[2] Wijffels M, Kirchhof C, Dorland R, Allessie M. Atrial fibrillation begets atrial fibrillation. A study in awake chronically instrumented goats. Circulation 1995;(92):19541968.

[3] Langley P, Bourke JP, Murray A. Frequency analysis of atrial fibrillation. In Proc.Int.Conf on Computers in Cardiology. 2000; 65-68.

[4] Stridh M, Sörmno L, Meurling C, Olsson B. Characterization of atrial fibrillation using the surface ECG: time-dependent spectral properties. IEEE Transactions on Biomedical Engineering 2001;48(1):19-27.
[5] Langley P, Rieta JJ, Stridh M, Millet J, Sörnmo L, Murray A. Comparison of atrial signal extraction algorithms in 12lead ECGs with atrial fibrillation. IEEE Transactions on Biomedical Engineering 2006;53(2):343-346.

[6] Slocum J, Sahakian A, Swiryn S. Diagnosis of atrial fibrillation from surface electrocardiogram based on computerdetected atrial activity. Journal of Electrocardiology 1992; 25(1):1-8.

[7] Rieta JJ, Castells F, Sánchez C, Zarzoso V. Atrial activity extraction for atrial fibrillation analysis using blind source separation. IEEE Transations on Biomedical Engineering 2004;51(7):1176-1186.

[8] Pincus SM. Approximate entropy (ApEn) as complexity measure. Chaos 1995;5(1):110-117.

[9] Richman JS, Moorman JR. Physiological time-series analysis using approximate entropy and sample entropy. Am J Physiol Heart Circ Physiol 2000;(278):H2039-H2049.

[10] Hornero R, Abasolo D, Jimeno N, I SC, Poza J, Aboy M. Variability, regularity and complexity of time series generated by schizophrenic patients and control subjects. IEEE Trans Biomed Eng 2006;53(2):210-218.

[11] Vayá C, Rieta JJ, Alcaraz R, Sánchez C, Cervigón R. Prediction of atrial fibrillation termination by approximate entropy in the time-frequency domain. In Proc.Int.Conf on Computers in Cardiology. 2006; 589-592.

[12] Vayá C, Rieta JJ, Sánchez C, Moratal D. Convolutive blind source separation algorithms applied to the electrocardiogram of atrial fibrillation: Study of performance. IEEE Transactions on Biomedical Engineering 2007;54(8):1530 1533.

[13] Bartels R, Beatty, Barsky B. An Introduction to Splines for Use in Computer Graphics and Geometric Modeling. Morgan Kauffman, 1987.

[14] Nielson E, Ahlberg JH, , Walsh J. The Theory of Splines and Their Applications. Academic Press: New York, 1967.

[15] Botteron GW, Smith JM. Quantitative assessment of the spatial organization of atrial fibrillation in the intact human heart. Circulation 1996;93:513-518.

Address for correspondence:

Carlos Vayá

Innovation in Bioengineering.

Universidad de Castilla-La Mancha.

Camino del Pozuelo, s/n, 16071, Cuenca.

carlos.vaya@uclm.es 